\title{
Diffusion weighted MRI in chronic viral hepatitis: correlation between ADC values and histopathological scores
}

\author{
Mehmet Ruhi Onur • Ahmet Kursad Poyraz • \\ Pinar Gundogan Bozdag • Semen Onder • Cem Aygun
}

Received: 15 December 2012 /Revised: 10 April 2013 /Accepted: 16 April 2013 /Published online: 11 May 2013

(C) The Author(s) 2013. This article is published with open access at Springerlink.com

\begin{abstract}
Objective To investigate the utility of apparent diffusion coefficient (ADC) measurement in the diagnosis of chronic viral hepatitis $(\mathrm{CVH})$ and correlation between $\mathrm{ADC}$ values and histopathologic severity of $\mathrm{CVH}$.

Materials and methods The ADC values of liver parenchyma on diffusion-weighted magnetic resonance imaging (DWMRI) were measured in 50 patients with a history of CVH and 51 healthy subjects at $b 100, b 600$ and $b 1,000$ gradients. Comparison between mean ADC values of the $\mathrm{CVH}$ and control groups and correlation results between $\mathrm{ADC}$ values and necroinflammation and fibrosis scores in $\mathrm{CVH}$ were obtained.

Results Mean ADC values of CVH patients were significantly lower than mean $\mathrm{ADC}$ values of the control group at $b 100$ and $b 600$ gradients $(P<0.05)$. There was no significant difference between the $\mathrm{CVH}$ and control groups at the $b 1,000$ gradient $(P>0.05)$. No significant correlation was found between $\mathrm{ADC}$ values and histopathologic scores of $\mathrm{CVH}(P>0.05)$.

Conclusion ADC values obtained at the $b 100$ and $b 600$ gradients can be used to distinguish between the liver parenchyma of $\mathrm{CVH}$ and healthy subjects. ADC measurement
\end{abstract}

\footnotetext{
M. R. Onur · A. K. Poyraz • P. G. Bozdag

Department of Radiology, University of Firat Faculty of Medicine, Elazig, Turkey

\section{S. Onder}

Department of Pathology, University of Istanbul Faculty of Medicine, Istanbul, Turkey

\section{Aygun}

Department of Gastroenterology,

University of Medipol Faculty of Medicine, Istanbul, Turkey
}

\section{R. Onur $(\bowtie)$}

Firat Universitesi, Hastanesi rektorluk Kampusu,

23119 Elazig, Turkey

e-mail: ruhionur@yahoo.com was not found to be useful for estimation of the degree of necroinflammation and fibrosis in $\mathrm{CVH}$.

Teaching Points

- In chronic viral hepatitis apparent coefficient values are decreased in the liver

- There is no correlation between ADC values and histopathologic severity of $\mathrm{CVH}$

- DW images obtained at low $b$ values have more ability to demonstrate an ADC decrease in viral hepatitis

Keywords Chronic viral hepatitis · Fibrosis · Diffusionweighted MRI $\cdot$ ADC measurements

\section{Introduction}

Chronic viral hepatitis $(\mathrm{CVH})$ is one of the most common causes of hepatic fibrosis, cirrhosis, portal hypertension and hepatocellular carcinoma [1]. Early detection of parenchymal fibrosis has important clinical implications for $\mathrm{CVH}$ since antiviral treatment can increase patient survival and reduce the need for liver transplantation [2,3]. A liver biopsy is the gold standard method for assessing changes in the parenchyma in CVH. However, it is associated with a risk of complications and may result in death in $0.018 \%$ of patients [4]. Determination of the severity of CVH by noninvasive imaging is a major topic discussed in studies of liver imaging [5]. Diffusion-weighted magnetic resonance imaging (DWMRI) is a functional MRI technique that takes advantage of the diffusion properties of water molecules in biological tissues. The microscopic movement of water molecules in biological tissues can be measured by apparent diffusion coefficient (ADC) values derived from DWMRI. The ADC values were reported to be inversely related to the degree of severity of CVH $[6,7]$.

The histopathological features of CVH consist of necroinflammation and fibrosis, which represent the activity 
and stage of the disease, respectively. The severity of necroinflammation and fibrosis can be assessed by histopathological scoring methods. The correlations between ADC values of different diffusion gradients (low, intermediate or high level) and the subtypes of histopathological scores (necroinflammation and fibrosis) have not been definitely determined [7-9].

In this study, the aim was to investigate the utility of the ADC measurement at low ( $b$ 100), intermediate $(b 600)$ and high $(b 1,000)$ levels of diffusion gradients in estimating the histopathological severity of CVH. In order to perform this study, we determined the correlations between the ADC values and the histopathological scores of liver necroinflammation and fibrosis in patients with $\mathrm{CVH}$.

\section{Materials and methods}

\section{Study group}

This prospective study was performed between June 2009 and May 2010. Fifty patients (23 female, 27 male; mean age: 44.46, age range: 21-69) with a history of CVH (32 hepatitis $\mathrm{B}$ and 18 hepatitis $\mathrm{C}$ ) underwent DWMRI prior to and on the same day of a liver parenchyma biopsy, and these subjects formed the CVH group. Fifty-one patients without a history of liver disease (25 female, 26 male; mean age: 42.24, age range: 18-74) who had undergone conventional and DWMRI for reasons other than liver disease at the time of this study were assigned to the control group. Subjects who had previously unrecognised, suspicious focal liver lesions $(n: 6)$ were excluded from the $\mathrm{CVH}$ group. Control subjects with a diffuse/focal signal decrease ( $n: 4)$ due to steatosis and/or iron deposition on T1-weighted out-of-phase and T2weighted images, respectively, were excluded from the control group. The study protocol was approved by the institutional review board and informed consent was obtained from all participants.

\section{MRI protocol}

MRI examinations were performed with the Signa Excite $1.5 \mathrm{~T}$ system (GE Healthcare, Milwaukee, WI, USA) with a four-channel torso body coil. The DW images were acquired with $b 100(0-100), b 600(0-600)$ and $b \quad 1,000(0-1,000) \mathrm{s} / \mathrm{mm}^{2}$ gradients. Before DWI was conducted, breath hold, axial 3D gradient-echo T1-weighted sequence, 2D gradient-echo T1 in-phase and out-phase, axial respiratory-triggered turbo spin-echo T2-weighted sequence with fat saturation images were obtained. All DWIs were acquired in the transverse plane using single-shot echo-planar spin echo sequences obtained when the subject was breathing normally. The parameters of the DWMRI were as follows: TR: $8,000 \mathrm{~ms}$; TE: $80 \mathrm{~ms}$; FOV: $30 \mathrm{~cm} \times 30 \mathrm{~cm}$; number of excitations: 2; matrix size: $128 \times 128$; section thickness: $5 \mathrm{~mm}$; slice number: 72 ; intersection gap: none; acquisition time ( 3 gradients): $96 \mathrm{~s}$. The array spatial sensitivity encoding technique (ASSET) was used as the parallel imaging technique with a factor of 2 . Diffusion-sensitising gradients were applied in three orthogonal directions: frequency-encoding $(x)$, phase-encoding $(y)$ and section-select $(z)$.

\section{Analysis of images}

ADC measurements were performed on colour-coded ADC maps automatically after calculating the diffusion difference between each gradient ( $b$ 100, 600 and 1,000) and the $b 0$ gradient. The ADC values of the liver parenchyma were obtained in patients with $\mathrm{CVH}$ and in the control group by taking measurements in three equally sized, circular regions of interest (ROIs) in segment 6 of the right lobe of the liver at $b 100, b$ 600 and $b 1,000$ gradients using specialised software (OsiriX Medical Imaging Software, Atlanta, GA, USA). The radiologist who measured the ADC values was blinded to the histopathologic results and clinical histories of the patients. The ROIs were placed on one slice/location on the DW images and then were inserted onto the corresponding location on the ADC map using the copy and paste function. The size of the ROIs in all patients was equal and was set at $1 \mathrm{~cm}^{2}$. The ROI was placed far away from visible vascular structures and at least $1 \mathrm{~cm}$ away from the Glissonian capsule. The ROIs were manually and carefully positioned to be in the same region in the three corresponding DW images performed at different $b$ factors. The final ADC for each subject that was used for statistical analysis was the average of the three ADC values obtained from the right lobe of the liver.

Histopathological analysis

Biopsy specimens were obtained from the parenchyma of segment 6 in the right lobe of the liver with an 18gauge core biopsy needle (Bard MaxCore Disposable Core Biopsy Instrument, Tempe, AZ, USA) under ultrasound guidance. The mean length of the specimens was $21.2 \mathrm{~mm}$. The number of specimens obtained from the patients ranged from 1 to 3 . The specimens were fixed in formalin and stained with hematoxylin-eosin, a stain for reticulin, and a Masson trichrome stain. The stage of liver fibrosis and the grade of the inflammatory changes 


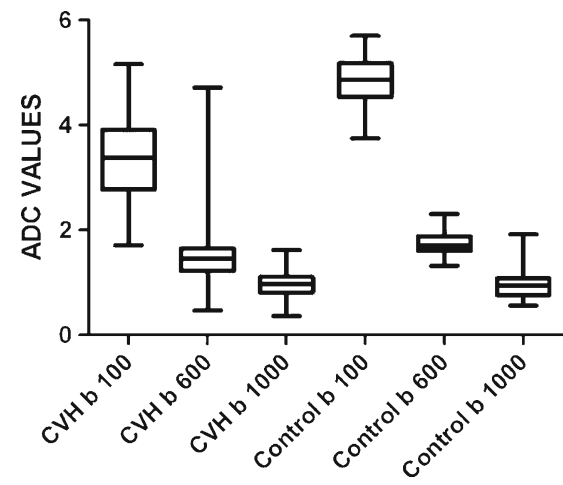

Fig. 1 Boxplot graphic demonstrates ADC values of patients in the $\mathrm{CVH}$ and control group at $b 100, b 600$ and $b 1,000$ gradients

were determined by two experienced pathologists using the Ishak scoring method. The pathologists were blinded to the MRI results and serologic tests. The subcategories of necroinflammation (which represented the activity of the disease) with the corresponding range of scores based on the Ishak method were as follows: periportal hepatitis (piecemeal necrosis) (score range: $0-4$ ), confluent necrosis (score range: 0-6), focal necrosis (score range: $0-4$ ) and portal inflammation (score range: $0-4$ ). The severity of liver fibrosis (which represented the stage of the disease) ranged from 1 to 5 using the Ishak fibrosis scoring method.

\section{Statistical analysis}

All ADC values were defined as mean $\pm \mathrm{SD}$. The mean ADC values of the $b 100, b 600$ and $b 1,000$ diffusion gradients were calculated. Comparisons between the mean $\mathrm{ADC}$ values of the $\mathrm{CVH}$ and control groups at the $b 100, b 600$ and $b 1,000$ gradients were performed using unpaired Student $t$ tests. The correlations between the ADC values of the three diffusion gradients and the Ishak scores of necroinflammation and fibrosis were determined using Pearson's correlation test. Statistical analysis was performed using Prism 5.0 software (Graphpad software, Inc., San Diego, CA, USA). The differences in the ADC values were considered to be statistically significant when the $P$ value was $<0.05$.

Table 1 Mean ADC values $\left(\times 10-{ }^{3} \mathrm{~mm}^{2} / \mathrm{s}\right)$ of liver parenchyma in the $\mathrm{CVH}$ and control group and at $b 100, b 600$ and $b 1,000$ gradients

\begin{tabular}{llll}
\hline Patients & $b 100$ & $b 600$ & $b 1,000$ \\
\hline CVH & $3.35 \pm 0.85$ & $1.50 \pm 0.67$ & $0.95 \pm 0.24$ \\
Control group & $4.80 \pm 0.50$ & $1.71 \pm 0.21$ & $0.99 \pm 0.32$ \\
Statistical significance & $P<0.0001$ & $P=0.043$ & $P=0.52$ \\
\hline
\end{tabular}

\section{Results}

There was no significant difference in the mean age of the CVH group $(44.46 \pm 13.19)$ and the control group (42.24 \pm 12.44$)(P>0.05)$. The ADC values of the liver parenchyma in the $\mathrm{CVH}$ and control group ranged from $0.36-4.16 \mathrm{~mm}^{2} / \mathrm{s}$ and $0.76-5.60 \mathrm{~mm}^{2} / \mathrm{s}$, respectively (Fig. 1). The mean $\mathrm{ADC}$ values of the $\mathrm{CVH}$ group were significantly lower than the mean ADC values of the control group at the $b 100(P<0.0001)$ and $b 600$ gradients $(P=0.043)$ (Table 1) (Figs. 1 and 2). There was no significant difference between the mean ADC values of the patients in the $\mathrm{CVH}$ and the control group at the $b 1,000$ gradient $(P=0.52)$ (Table 1) (Fig. 3).

\section{a}

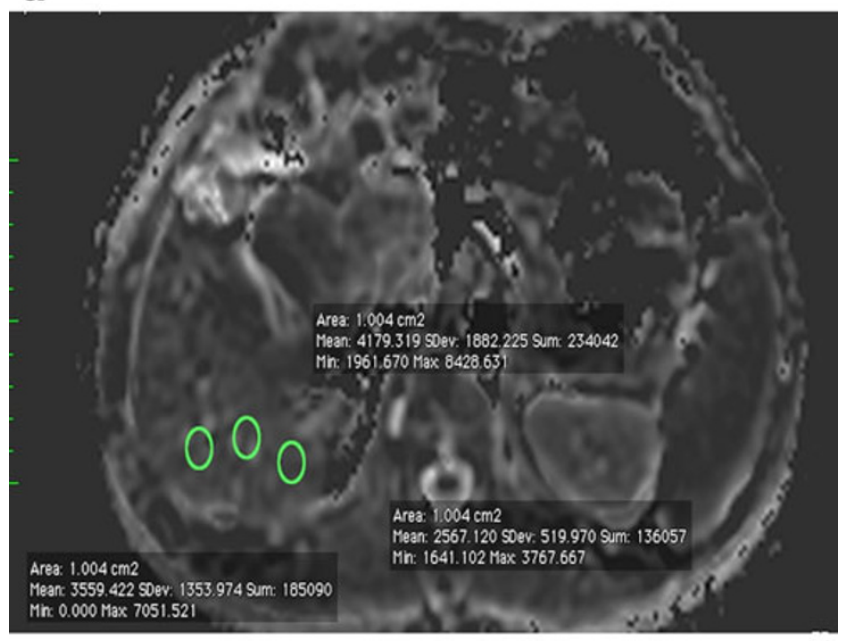

b

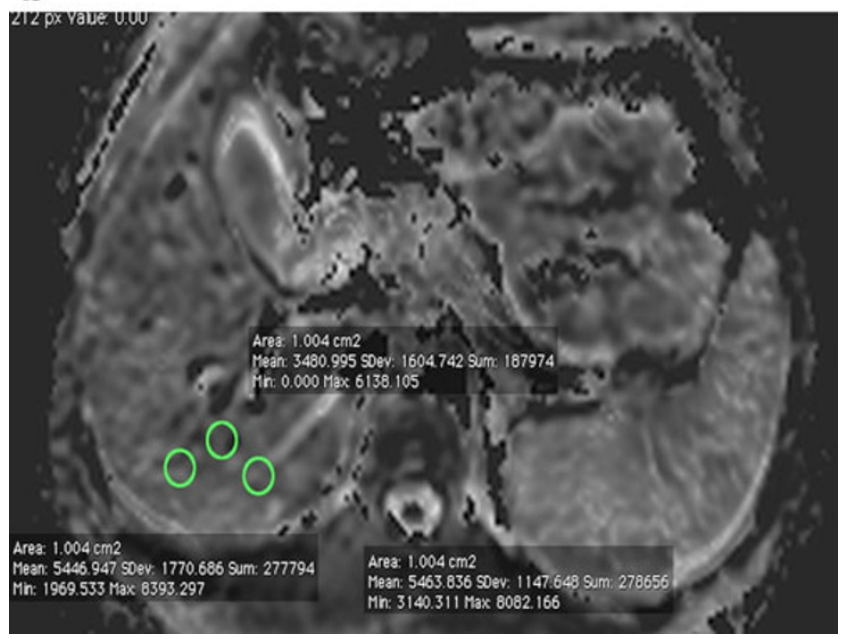

Fig. 2 ADC measurements at the $b 100$ gradient. Mean ADC value of liver parenchyma in a patient with $\mathrm{CVH}\left(3.43 \times 10-^{3} \mathrm{~mm}^{2} / \mathrm{s}\right)$ (a) was significantly lower than the mean ADC value $\left(4.79 \times 10-^{3} \mathrm{~mm}^{2} / \mathrm{s}\right)$ of a patient in the control group (b) 
Fig. 3 ADC measurement at $b$ 1,000 gradient. Mean ADC value of liver parenchyma $\left(0.95 \times 10-3 \mathrm{~mm}^{2} / \mathrm{s}\right)($ a) $)$ is not significantly lower than in patients in the control group $\left(0.99 \times 10-{ }^{3} \mathrm{~mm}^{2} / \mathrm{s}\right)(\mathbf{b})$

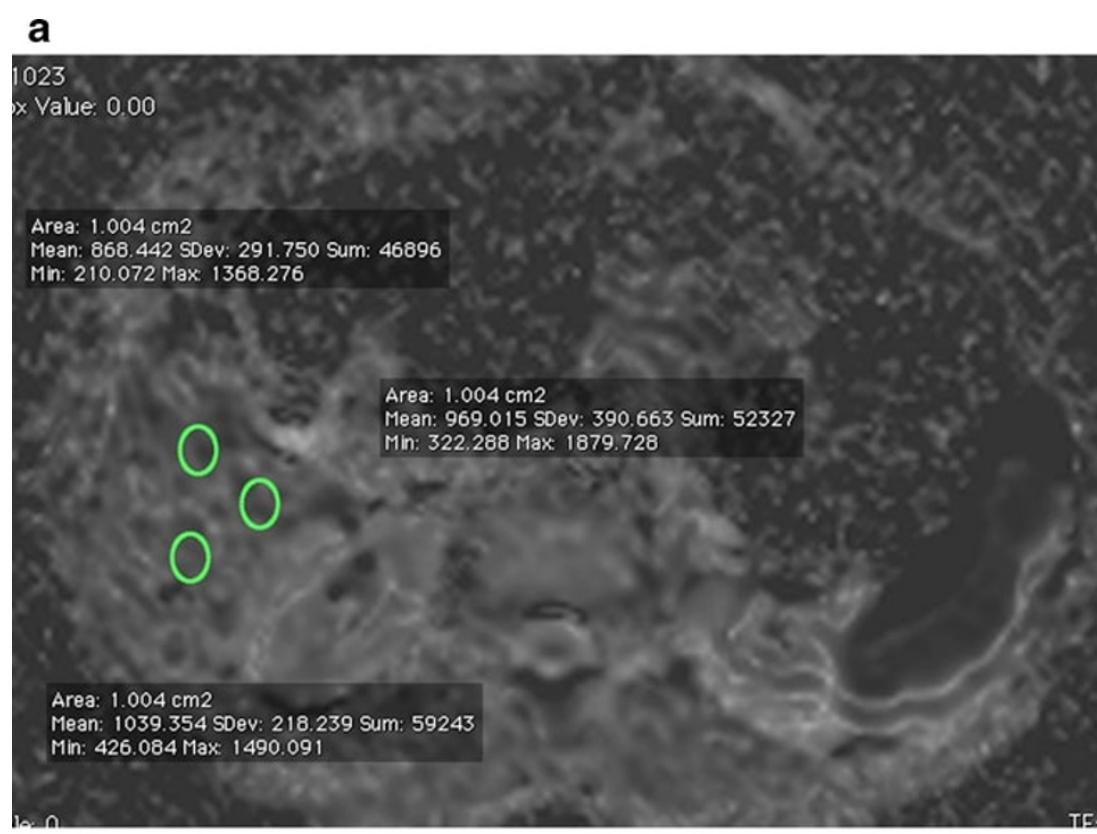

b

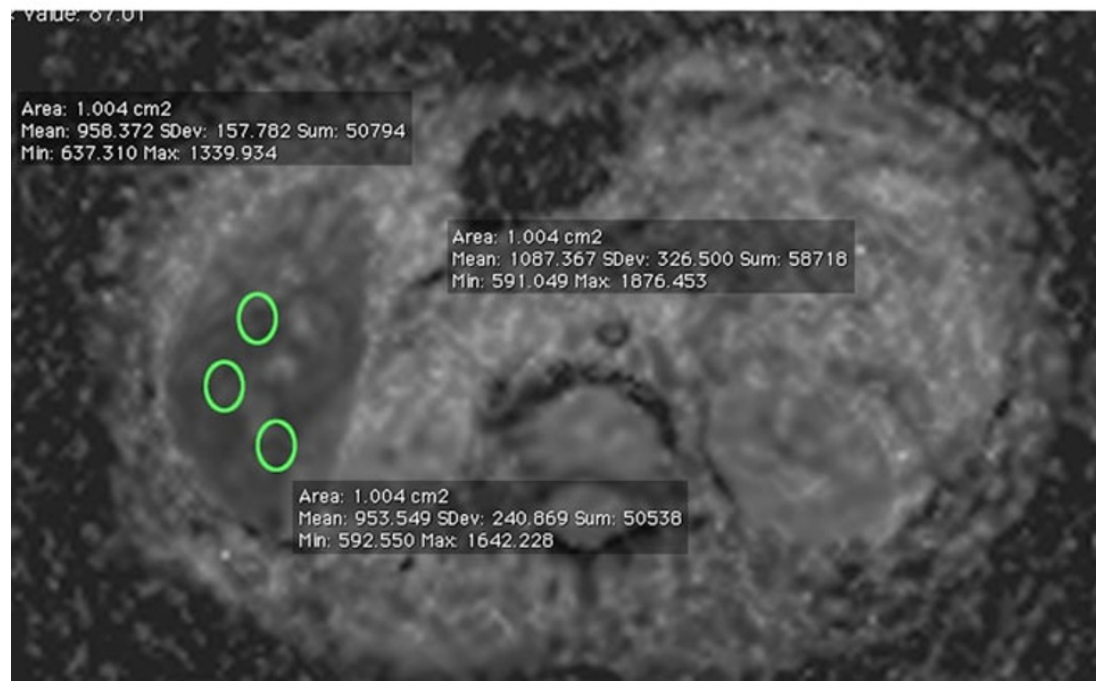

The necroinflammation and fibrosis scores of liver biopsy specimens ranged from 4-16 and 1-5, respectively. Table 2 lists the subcategories of necroinflammation and the corresponding number and mean ADC value of the patients. Table 3 lists the fibrosis scores and the corresponding number and mean ADC values of the patients. There was no significant correlation found between the ADC values of the $b 100, b 600$ and $b 1,000$ gradients and the Ishak scores of necroinflammation and fibrosis (Table 4).

\section{Discussion}

Chronic viral hepatitis is one of the major causes of liver failure. A patient's rate of progression to fibrosis determines the prognosis and the need for treatment [6]. The main pathophysiological mechanisms of the development and progression of parenchymal changes in $\mathrm{CVH}$ are inflammatory cell infiltration, necrosis, fibrosis and decreased perfusion of liver parenchyma [10]. Early diagnosis of $\mathrm{CVH}$ is essential to prevent or delay endstage chronic parenchymal liver disease. Ultrasound elastography, diffusion-weighted and perfusion-weighted MRI, MR elastography, chemical shift-based fat-water separation and MR spectroscopy are widely used imaging techniques for the assessment of chronic liver disease [5]. One proposed, noninvasive tool for the assessment of the severity of CVH is ADC measurement on DWMRI.

The ADC value in biological tissues represents water proton motion, which is mainly determined by both the 
Table 2 Distribution of mean ADC values at $b 100, b 600$ and $b 1,000$ gradients among necroinflammation scores of $\mathrm{CVH}$

\begin{tabular}{lllll}
\hline $\begin{array}{l}\text { Necroinflammation } \\
\text { score }\end{array}$ & $\begin{array}{l}\text { Number } \\
\text { of patients }\end{array}$ & $b 100$ & $b 600$ & $b 1,000$ \\
\hline 4 & 2 & 3.43 & 1.42 & 1.12 \\
5 & 4 & 3.23 & 1.30 & 0.91 \\
6 & 2 & 3.20 & 1.49 & 1.07 \\
7 & 5 & 3.15 & 1.24 & 1.03 \\
8 & 4 & 3.14 & 1.26 & 0.90 \\
9 & 4 & 3.20 & 1.19 & 0.75 \\
10 & 8 & 2.86 & 1.27 & 0.98 \\
11 & 7 & 2.90 & 1.16 & 0.91 \\
12 & 7 & 2.67 & 1.13 & 0.84 \\
13 & 5 & 2.33 & 1.15 & 0.93 \\
14 & 1 & 2.50 & 1.18 & 0.88 \\
15 & 0 & - & - & - \\
16 & 1 & 2.47 & 0.94 & 0.87 \\
17 & 0 & - & - & - \\
18 & 0 & - & - & - \\
\hline
\end{tabular}

true water diffusion and capillary perfusion. Low diffusion gradients yield high ADC values in comparison to high diffusion gradients [11]. The ADC values at low diffusion gradients represent not only the diffusion, but also the perfusion properties of tissues. The ADC values at high gradients represent only molecular diffusion and tend to be much lower than ADC values obtained at low diffusion gradients. In previous studies, lower ADC values were obtained in the liver parenchyma of patients with $\mathrm{CVH}$ compared to healthy subjects $[6,7,9]$. The underlying reason for the decreased $\mathrm{ADC}$ values in $\mathrm{CVH}$ remains controversial. Decreased ADC values may be the result of decreased diffusion of water molecules secondary to increased connective tissue in the liver, constricted sinusoids and decreased blood flow [12]. Diminished liver perfusion in patients with $\mathrm{CVH}$ and cirrhosis has been detected using MR perfusion studies [13]. Decreased perfusion of the liver has been suggested to be the primary reason for the apparent

Table 3 Distribution of mean ADC values at $b 100, b 600$ and $b 1,000$ gradients among fibrosis scores of $\mathrm{CVH}$

\begin{tabular}{lllll}
\hline Fibrosis score & No. of patients & $b 100$ & $b 600$ & $b 1,000$ \\
\hline 1 & 6 & 3.15 & 1.34 & 1.01 \\
2 & 22 & 3.15 & 1.27 & 0.92 \\
3 & 11 & 2.94 & 1.30 & 0.98 \\
4 & 6 & 2.87 & 1.28 & 0.90 \\
5 & 5 & 2.90 & 1.20 & 0.85 \\
\hline
\end{tabular}

decrease in diffusion. An animal study revealed that rats with hepatic fibrosis had reduced ADC values in vivo but not when DWI was performed ex vivo [14]. Similarly, in a study conducted with cirrhotic live dogs, the $\mathrm{ADC}$ values of the cirrhotic lobes were lower than in the normal lobes; however, the differences between lobes in the ADC values disappeared after portal vein clamping [15]. In addition, a study by Luciani et al. suggested that the restricted diffusion observed in patients with cirrhosis reflects diminished capillary perfusion and, to a much lesser extent, decreased pure molecular diffusion [16]. Since lower $b$ values are more sensitive to the effects of perfusion than higher diffusion gradients, it has been suggested that they are of more use in determining the hepatic fibrosis stage [7]. In our study, we found significant differences between the mean $\mathrm{ADC}$ values in $\mathrm{CVH}$ patients and healthy subjects only at the $b 100$ and $b 600$ gradients. It is likely that the difference in the liver perfusion between healthy subjects and $\mathrm{CVH}$ patients led to the significant difference in the ADC values of two groups at the $b$ 100 and $b 600$ gradients in our study. The mean ADC value of the $\mathrm{CVH}$ at the $b 1,000$ gradient was lower than the control group but there was no significant difference. We suggest that the lack of a significant difference in the ADC values at the $b 1,000$ gradient in the $\mathrm{CVH}$ and control group is due to the fact that the ADC measurement at the $b 1,000$ gradient is not affected by perfusion. This result is in concordance with previous studies, which also emphasise the importance of decreased perfusion in CVH [14].

The histopathologic evaluation of $\mathrm{CVH}$ consists of scoring necroinflammation and fibrosis in the liver parenchyma. The Ishak method was reported to be a useful tool for performing pathological research and for helping the pathologist to take note of all aspects of viral injury including periportal hepatitis, confluent necrosis, focal lytic necrosis, portal inflammation and fibrosis [10]. Different results have been obtained in previous studies regarding the correlation between ADC values and necroinflammatory activity or fibrosis scores [7,9]. In the earlier studies, the ADC values obtained at low diffusion gradients $\left(b<250 \mathrm{~s} / \mathrm{mm}^{2}\right)$ were found to be negatively correlated with fibrosis scores but not with necroinflammation scores [7, 8]. Later studies found that there was a negative correlation between ADC values at both low and high diffusion gradients and necroinflammation scores [9, 12]. These studies also suggested that in addition to fibrosis, the ADC values might reflect the intensity of inflammation or necrosis and decrease when there were alterations in tissue structure $[9,12]$. However, Boulanger et al. used DWI at $b$ values of $50-250 \mathrm{~s} / \mathrm{mm}^{2}$ to examine 18 hepatitis $\mathrm{C}$ virus patients and 10 control 
Table 4 Correlation results between ADC values of $b 100, b 600$ and $b 1,000$ gradients and Ishak scores of necroinflammation and fibrosis in CVH

\begin{tabular}{|c|c|c|c|c|c|c|}
\hline & \multicolumn{2}{|l|}{ b 100} & \multicolumn{2}{|l|}{$b 600$} & \multicolumn{2}{|l|}{$b 1,000$} \\
\hline & $\mathrm{PC}$ & $P$ & $\mathrm{PC}$ & $P$ & $\mathrm{PC}$ & $P$ \\
\hline Necroinflammation & -0.025 & 0.877 & -0.109 & 0.462 & -0.124 & 0.397 \\
\hline Fibrosis & -0.050 & 0.755 & -0.025 & 0.867 & -0.119 & 0.415 \\
\hline
\end{tabular}

$P C$ Pearson's correlation

$P$ significance

subjects and found no significant correlation between the ADC values and the inflammation or fibrosis scores [8]. Similarly, Soylu et al. calculated the ADC values using measurements from four segments of liver lobes at $b$ values of 0 , 500 and $1,000 \mathrm{~s} / \mathrm{mm}^{2}$ and did not find any correlation between the ADC values and liver fibrosis scores at the $b 500$ and $b$ 1,000 diffusion gradients [17]. In our study, we used $b 100, b$ 600 and $b$ 1,000 gradients representing low, intermediate and high level diffusion gradients, respectively, in order to determine which gradient would be most effective in obtaining a correlation between ADC values and histopathological scores of CVH. It was found that the ADC values obtained at the $b$ $100, b 600$ and $b 1,000$ gradients were not significantly correlated with necroinflammation and fibrosis scores (Table 4). In general, the ADC values decreased as the necroinflammation and fibrosis scores increased, but this relationship was not statistically significant. Also, when the ADC values of each necroinflammatory and fibrosis score were assessed, it was found that there were overlapping ADC values, which is a finding reported in other studies as well [18]. Thus, our study suggests that ADC measurements at low, intermediate and high diffusion gradients cannot be used to evaluate the severity of $\mathrm{CVH}$ as reliably as the histopathological scores.

The present study has several limitations. First, although the ROIs were carefully positioned to be in the same region between two corresponding images, possible differences in the ROI position may have a slight effect on the ADC values. Second, the location of the ROIs used to calculate the ADC value were not the same as the areas selected for liver biopsy. To overcome this limitation we intended to measure ADC values in segment 6 of the right liver lobe where the biopsy specimens were obtained. In this study, we preferred to use $b$ values of $0,100,600$ and 1,000 and measured the ADC values at the diffusion strengths between $b$ $0-100, b$ 0-600 and $b$ 0-1,000. Measurement of ADC values by using multiple $b$ values in different combinations increases the diagnostic accuracy of ADC values in biological tissues [19]. The ADC measurement at the diffusion gradients between $b$ 100-600, $b$ 100-1,000 and $b$ 600-1,000 would be more helpful to understand the utility of ADC values in differentiation between $\mathrm{CVH}$ and normal liver parenchyma in our study. Measurement of ADC values with monoexponential function is another limitation of our study. The monoexponential method is a simple method of ADC measurement and widely available on most MRI scanners; however, it is influenced by micropapillary perfusion. The signal attenuation of body tissues on DWMRI manifests with biexponential behaviour [20]. Although monoexponential function has been used for ADC measurements in MRI workstations, the biexponential method has been widely accepted as a reliable method, and ADC values obtained with the biexponential method are more likely to represent diffusion properties of tissues in comparison to the monoexponential method. We used the monoexponential method for ADC measurements in our study since our MRI system workstation reveals ADC values automatically with the monoexponential function. As a last limitation, measurement of ADC values at high diffusion gradients such as the $b 1,000$ gradient may cause variable ADC values secondary to decreased signal-to-noise ratios in these gradients [11].

In conclusion, we found that the ADC values obtained at the $b 100$ and $b 600$ gradients can be used to distinguish between the liver parenchyma of CVH patients and healthy subjects. However, the ADC measurement was not found to be a useful tool for evaluating the degree of necroinflammation and fibrosis in CVH. Our findings support the results of previous studies that suggest that liver perfusion is a major cause for the decrease in ADC values in $\mathrm{CVH}$.

\section{Funding and conflict of interest None}

Open Access This article is distributed under the terms of the Creative Commons Attribution License which permits any use, distribution, and reproduction in any medium, provided the original author(s) and the source are credited. 


\section{References}

1. Afdhal NH, Nunes D (2004) Evaluation of liver fibrosis: a concise review. Am J Gastroenterol 99:1160-1174

2. Lawrence SP (2000) Advances in the treatment of hepatitis C. Adv Intern Med 45:65-105

3. Buti M, San Miguel R, Brosa M, Cabases JM, Medina M, Angel Casado $\mathrm{M}$ et al (2005) Estimating the impact of hepatitis $\mathrm{C}$ virus therapy on future liver-related morbidity, mortality and costs related to chronic hepatitis C. J Hepatol 42:639-645

4. Wong JB, Bennet WG, Koff RS, Pauker SG (1998) Pretreatment evaluation ofchronic hepatitis C: risks, benefits, and costs. JAMA 280:2088-2093

5. Taouli B, Ehman RL, Reeder SB (2009) Advanced MRI methods for assessment of chronic liver disease. AJR Am J Roentgenol 193(1):14-27

6. Girometti R, Furlan A, Bazzocchi M, Soldano F, Isola M, Toniutto $P$ et al (2007) Diffusion-weighted MRI in evaluating liver fibrosis: a feasibility study in cirrhotic patients. Radiol Med 112(3):394408

7. Koinuma M, Ohashi I, Hanafusa K, Shibuya H (2005) Apparent diffusion coefficient measurements with diffusion-weighted magnetic resonance imaging for evaluation of hepatic fibrosis. J Magn Reson Imaging 22(1):80-85

8. Boulanger Y, Amara M, Lepanto L, Beaudoin G, Nguyen BN, Allaire $G$ et al (2003) Diffusion-weighted MR imaging of the liver of hepatitis C patients. NMR Biomed 16:132-136

9. Lewin M, Poujol-Robert A, Boëlle PY, Wendum D, Lasnier E, Viallon $M$ et al (2007) Diffusion-weighted magnetic resonance imaging for the assessment of fibrosis in chronic hepatitis C. Hepatology 46:658-665

10. Theise ND (2007) Liver biopsy assessment in chronic viral hepatitis: a personal, practical approach. Mod Pathol 20(Suppl 1):S3S14
11. Vermoolen MA, Kwee TC (2012) Nievelstein RA (2012) Apparent diffusion coefficient measurements in the differentiation between benign and malignant lesions: a systematic review. Insights Imaging 3(4):395-409

12. Taouli B, Chouli M, Martin AJ, Qayyum A, Coakley FV, Vilgrain V (2008) Chronic hepatitis: role of diffusionweighted imaging and diffusion tensor imaging for the diagnosis of liver fibrosis and inflammation. J Magn Reson Imaging 28(1):89-95

13. Annet L, Materne R, Danse E, Jamart J, Horsmans Y, Van Beers BE (2003) Hepatic flow parameters measured with MR imaging and Doppler US:correlations with degree of cirrhosis and portal hypertension. Radiology 229:409-414

14. Annet L, Peeters F, Abarca-Quinones J, Leclercq I, Moulin P, Van Beers BE (2007) Assessment of diffusion-weighted MR imaging in liver fibrosis. J Magn Reson Imaging 25:122-128

15. Yang ZH, Ye XH, Tan Y, Zhang M, Zhou MZ, Xie JX et al (2006) Evaluation of cirrhotic liver with perfusion-weighted magnetic resonance imaging: a preliminary experimental study in animal models with half-liver cirrhosis. Chin Med Sci J 21:252-257

16. Luciani A, Vignaud A, Cavet M, Nhieu JT, Mallat A, Ruel L et al (2008) Liver cirrhosis: intravoxel incoherent motion MR imaging - pilot study. Radiology 249:891-899

17. Soylu A, Kiliçkesmez O, Poturoğlu S, Dolapçioğlu C, Serez K, Sevindir I et al (2010) Utility of diffusion-weighted MRI for assessing liver fibrosis in patients with chronic active hepatitis. Diagn Interv Radiol 16(3):204-208

18. Sandrasegaran K, Akisik FM, Lin C, Tahir B, Rajan J, Saxena R et al (2009) Value of diffusion-weighted MRI for assessing liver fibrosis and cirrhosis. AJR Am J Roentgenol 193(6):1556-1560

19. Chandarana H, Taouli B (2010) Diffusion and perfusion imaging of the liver. Eur J Radiol 76(3):348-358

20. Guiu B, Cercueil JP (2011) Liver diffusion-weighted MR imaging: the Tower of Babel? Eur Radiol 21(3):463-467 